Article

\title{
Tumor Long Interspersed Nucleotide Element-1 (LINE-1) Hypomethylation in Relation to Age of Colorectal Cancer Diagnosis and Prognosis
}

\author{
Naohiko Akimoto 1,2 ${ }^{1}$, Melissa Zhao ${ }^{1}$, Tomotaka Ugai ${ }^{1,3}$, Rong Zhong 1,3,4, Mai Chan Lau ${ }^{1}$, Kenji Fujiyoshi ${ }^{1}$, \\ Junko Kishikawa $^{1}$, Koichiro Haruki ${ }^{1}{ }^{\mathbb{D}}$, Kota Arima ${ }^{1}$, Tyler S. Twombly ${ }^{1}$, Xuehong Zhang ${ }^{5,6}$, \\ Edward L. Giovannucci 3,5,6, Kana Wu 3,5,6, Mingyang Song 6,7,8, Andrew T. Chan 5,7,8,9, Yin Cao 10,11,12, \\ Jeffrey A. Meyerhardt ${ }^{13}$, Kimmie Ng ${ }^{13}$, Marios Giannakis ${ }^{13,14,15}$, Juha P. Väyrynen 1,13,16,*(D), \\ Jonathan A. Nowak ${ }^{1}$ and Shuji Ogino $1,3,14,17, *$ (D)
}

\section{check for} updates

Citation: Akimoto, N.; Zhao, M.; Ugai, T.; Zhong, R.; Lau, M.C.; Fujiyoshi, K.; Kishikawa, J.; Haruki, K.; Arima, K.; Twombly, T.S.; et al. Tumor Long Interspersed Nucleotide Element-1 (LINE-1) Hypomethylation in Relation to Age of Colorectal Cancer Diagnosis and Prognosis. Cancers 2021, 13, 2016. https:/ / doi.org/10.3390/cancers13092016

Academic Editor: Luca Roncucci

Received: 15 March 2021

Accepted: 18 April 2021

Published: 22 April 2021

Publisher's Note: MDPI stays neutral with regard to jurisdictional claims in published maps and institutional affiliations.

Copyright: (c) 2021 by the authors. Licensee MDPI, Basel, Switzerland. This article is an open access article distributed under the terms and conditions of the Creative Commons Attribution (CC BY) license (https:// creativecommons.org/licenses/by/ $4.0 /)$.
1 Program in MPE Molecular Pathological Epidemiology, Department of Pathology, Brigham and Women's Hospital, Harvard Medical School, Boston, MA 02115, USA; nakimoto1@bwh.harvard.edu (N.A.); mzhao11@bwh.harvard.edu (M.Z.); tugai@bwh.harvard.edu (T.U.); zhongr@hust.edu.cn (R.Z.); mclau@bwh.harvard.edu (M.C.L.); fujiyoshi_kenji@med.kurume-u.ac.jp (K.F.); KISHIKAWAJ-SUR@h.u-tokyo.ac.jp (J.K.); haruki@jikei.ac.jp (K.H.); kouarima-gi@umin.ac.jp (K.A.); ttwombly@bwh.harvard.edu (T.S.T.); janowak@bwh.harvard.edu (J.A.N.)

2 Department of Gastroenterology, Nippon Medical School, Graduate School of Medicine, Tokyo 1138602, Japan

3 Department of Epidemiology, Harvard T.H. Chan School of Public Health, Boston 02115, MA, USA; egiovann@hsph.harvard.edu (E.L.G.); hpkwu@channing.harvard.edu (K.W.)

4 Department of Epidemiology and Biostatistics, School of Public Health, Tongji Medical College, Huazhong University of Science and Technology, Wuhan 430030, China

5 Channing Division of Network Medicine, Department of Medicine, Brigham and Women's Hospital and Harvard Medical School, Boston, MA 02115, USA; poxue@channing.harvard.edu (X.Z.); achan@mgh.harvard.edu (A.T.C.)

6 Department of Nutrition, Harvard T.H. Chan School of Public Health, Boston, MA 02115, USA; mis911@mail.harvard.edu

7 Clinical and Translational Epidemiology Unit, Massachusetts General Hospital and Harvard Medical School, Boston, MA 02114, USA

8 Division of Gastroenterology, Massachusetts General Hospital, Boston, MA 02114, USA

9 Department of Immunology and Infectious Diseases, Harvard T.H. Chan School of Public Health, Boston, MA 02115, USA

10 Division of Public Health Sciences, Department of Surgery, Washington University in St. Louis, St. Louis, MO 63110, USA; yin.cao@wustl.edu

11 Alvin J. Siteman Cancer Center, Washington University School of Medicine, St. Louis, MO 63110, USA

12 Division of Gastroenterology, Department of Medicine, Washington University School of Medicine, St. Louis, MO 63110, USA

13 Department of Medical Oncology, Dana-Farber Cancer Institute and Harvard Medical School, Boston, MA 02215, USA; jeffrey_meyerhardt@dfci.harvard.edu (J.A.M.); kimmie_ng@dfci.harvard.edu (K.N.); marios_giannakis@dfci.harvard.edu (M.G.)

14 Broad Institute of MIT and Harvard, Cambridge, MA 02142, USA

15 Department of Medicine, Brigham and Women's Hospital, Harvard Medical School, Boston, MA 02115, USA

16 Cancer and Translational Medicine Research Unit, Medical Research Center Oulu, Oulu University Hospital, and University of Oulu, 90220 Oulu, Finland

17 Cancer Immunology and Cancer Epidemiology Programs, Dana-Farber Harvard Cancer Center, Boston, MA 02215, USA

* Correspondence: juha.vayrynen@oulu.fi (J.P.V.); sogino@bwh.harvard.edu (S.O.); Tel.: +358-46-923-6405 (J.P.V.); +1-617-732-5500 (S.O.)

Simple Summary: The rising incidence of early-onset cancers diagnosed before 50 years in many body sites (including the colorectum) is a growing concern. Despite the many studies of early-onset colorectal cancer, the characteristics of colorectal cancer diagnosed at age 50 or slightly after (close to age 50) have not been adequately examined. Although epigenetic alterations are considered to play a role in early-onset colorectal cancer, the association of LINE-1 hypomethylation (that reflects global DNA hypomethylation) with the age of colorectal cancer diagnosis has not been thoroughly clarified. Using a database of 1356 colorectal cancers, we found that tumor LINE-1 hypomethylation was 
increasingly more common with decreasing age of colorectal cancer diagnosis and was associated with higher colorectal cancer-specific mortality. Our findings support the "age continuum" model that has substantial implications in research on cancers in not only the colorectum but also many other body sites.

Abstract: Evidence indicates the pathogenic role of epigenetic alterations in early-onset colorectal cancers diagnosed before age 50. However, features of colorectal cancers diagnosed at age 50-54 (hereafter referred to as "intermediate-onset") remain less known. We hypothesized that tumor long interspersed nucleotide element-1 (LINE-1) hypomethylation might be increasingly more common with decreasing age of colorectal cancer diagnosis. In 1356 colorectal cancers, including 28 early-onset and 66 intermediate-onset cases, the tumor LINE-1 methylation level measured by bisulfite-PCRpyrosequencing (scaled 0 to 100) showed a mean of 63.6 (standard deviation (SD) 10.1). The mean tumor LINE-1 methylation level decreased with decreasing age (mean 64.7 (SD 10.4) in age $\geq 70$, 62.8 (SD 9.4) in age 55-69, 61.0 (SD 10.2) in age 50-54, and 58.9 (SD 12.0) in age $<50 ; p<0.0001$ ). In linear regression analysis, the multivariable-adjusted $\beta$ coefficient ( $95 \%$ confidence interval (CI)) (vs. age $\geq 70)$ was $-1.38(-2.47$ to -0.30$)$ for age $55-69,-2.82(-5.29$ to -0.34$)$ for age $50-54$, and $-4.54(-8.24$ to -0.85$)$ for age $<50\left(P_{\text {trend }}=0.0003\right)$. Multivariable-adjusted hazard ratios $(95 \% \mathrm{CI})$ for LINE-1 methylation levels of $\leq 45,45-55$, and 55-65 (vs. $>65$ ) were 2.33 (1.49-3.64), 1.39 (1.05-1.85), and $1.29(1.02-1.63)$, respectively $\left(P_{\text {trend }}=0.0005\right)$. In conclusion, tumor LINE-1 hypomethylation is increasingly more common with decreasing age of colorectal cancer diagnosis, suggesting a role of global DNA hypomethylation in colorectal cancer arising in younger adults.

Keywords: carcinogenesis; colorectal neoplasms; epigenomics; genomic instability; long interspersed nuclear element; molecular pathology; retrotransposon; screening; transposable element; youngonset cancer

\section{Introduction}

The past few decades have witnessed a rising incidence of early-onset colorectal cancer, defined as colorectal cancer diagnosed before 50 years of age, in substantial parts of the world, particularly high-income countries [1-3]. The causes underlying this phenomenon remain unclear. The problem of the rising incidence of early-onset cancers in many body sites (including the colorectum) [4] has ranked as the top 2020 Provocative Question of the U.S. National Cancer Institute. Undoubtedly, there is a heightened interest in the biology and drivers of early-onset colorectal cancer $[5,6]$.

Most of the previous studies regarding early-onset colorectal cancer adopted the dichotomy of age $<50 \mathrm{vs}$. $\geq 50$ years despite the lack of robust biological reasons to use age 50 as a cut point [7]. Only few studies examined tumor molecular features in relation to young age at onset, using models beyond the simple dichotomy at age $50[8,9]$. Therefore, the characteristics of colorectal cancer diagnosed at age 50 or after (but close to age 50) have not been adequately studied. For convenience, we hereafter designate colorectal cancer diagnosed at age 50-54 as "intermediate-onset colorectal cancer".

Epidemiological evidence suggests associations between early-life exposures and colorectal cancer $[10,11]$. Exposures to certain exogenous (environmental) and endogenous factors may lead to cellular epigenetic alterations [12-14], which may play a role in tumor development in young age. Long interspersed nucleotide element-1 (LINE-1, also known as long interspersed nuclear element-1) constitutes approximately $17 \%$ of the human genome, and its methylation level is well correlated with the global DNA methylation status [15]. LINE-1 hypomethylation in colorectal cancer has been associated with younger age at diagnosis $[16,17]$ and higher mortality [18-20]. However, LINE-1 hypomethylation of colorectal carcinoma in various age groups has not been thoroughly investigated. Our primary hypothesis was that tumor LINE-1 hypomethylation might be most common in early-onset colorectal cancer patients under 50 , followed by those aged 
50-54 (intermediate-onset), and least common in those aged $\geq 70$. We also hypothesized that tumor LINE-1 hypomethylation might be associated with poor prognosis.

To test the primary hypothesis, we compared tumor LINE-1 methylation levels between age groups in a molecular pathological epidemiology database of 1356 colorectal cancer cases, including early-onset and intermediate-onset cases. We further assessed the relationship of age groups with tumor LINE-1 hypomethylation, controlling for lifestyle and clinical factors as well as other tumor molecular characteristics (microsatellite instability (MSI) status, CpG island methylator phenotype (CIMP), KRAS mutation, BRAF mutation, and PIK3CA mutation) in multivariate-adjusted linear regression analysis. In addition, we assessed the prognostic significance of the LINE-1 methylation level in multivariable Cox regression models.

\section{Materials and Methods}

\subsection{Study Population}

We utilized two large prospective cohort studies in the United States, namely, the Nurses' Health Study (with 121,700 women aged 30 to 55 years, followed up since 1976) and the Health Professionals Follow-up Study (with 51,529 men aged 40 to 75 years, followed up since 1986) [21] (Figure 1). In both cohorts, questionnaires were sent to participants to update information on their lifestyle factors and medical history, including diagnosis of colorectal cancer every two years. The response rate for each follow-up questionnaire was more than $90 \%$ for both cohorts. We used data on colorectal cancer family history, pack-years of smoking (using all available biennial questionnaires), and body mass index (using the latest available questionnaire before diagnosis). The National Death Index was used to identify unreported lethal cases of colorectal cancer. Participating physicians, who were blinded to exposure data, reviewed the medical records of identified colorectal carcinoma cases to confirm the disease diagnosis and to collect data on clinical characteristics (e.g., tumor size, tumor location, and the American Joint Committee on Cancer (AJCC) tumor, node, and metastases (TNM) classification). A single pathologist (S.O.) performed a centralized review of hematoxylin and eosin-stained tissue sections from all colorectal carcinoma cases blinded to other data [22]. Tumor differentiation was categorized as moderate or poor ( $>50 \%$ vs. $\leq 50 \%$ glandular area, respectively). As a result, we utilized a molecular pathological epidemiology database of 1356 colorectal cancer cases, which included 28 patients diagnosed before age 50 (early-onset cases) and 66 patients diagnosed at age 50-54 (herein referred to as "intermediate-onset" cases), with available tumor LINE-1 methylation data. On the basis of the colorectal continuum model, both colon and rectal cancers were included [23].

Informed consent was obtained from all study participants at enrollment. This study was approved by the institutional review boards of the Brigham and Women's Hospital and Harvard T.H. Chan School of Public Health (Boston, MA, USA), and those of participating registries as required.

\subsection{Assessment of LINE-1 Methylation Level}

We collected formalin-fixed paraffin-embedded (FFPE) tissue blocks from hospitals where participants underwent tumor resection. Hematoxylin and eosin-stained slides of the tumors were reviewed, followed by marking of tumor areas. To enrich DNA from neoplastic cells, only tumor areas were macrodissected for DNA extraction. In a validation experiment for the LINE-1 assay [24], approximately 500 cancer cells from 5 anonymized cases were collected by laser capture microdissection (LCM) using an LCM instrument (MDS Analytical Technologies, CA, USA). Specimens were suspended in $140 \mu \mathrm{L}$ of tissue lysate solution ( $\mathrm{pH} 8,1 \mathrm{mg} / \mathrm{mL}$ proteinase $\mathrm{K}, 100 \mathrm{mmol} / \mathrm{L}$ Tris, $\mathrm{pH} 8,10 \mathrm{mmol} / \mathrm{L}$ ethylenediaminetetraacetic acid, and $0.05 \mathrm{mg} / \mathrm{mL}$ tRNA) and incubated overnight at $50{ }^{\circ} \mathrm{C}$. The lysate was aliquoted into seven tubes (each containing $18 \mu \mathrm{L}$ of tissue lysate) and stored at $-20{ }^{\circ} \mathrm{C}$ till sodium bisulfite modification was performed. 


\section{Original cohorts}

NHS: Women enrolled in $1976(n=121,700)$

HPFS: Men enrolled in $1986(n=51,529)$

\begin{tabular}{|l|}
\hline Followed-up from 1980/1986 to 2016 \\
NHS $(n=87,998)$ \\
HPFS $(n=47,344)$ \\
\hline $\begin{array}{l}\text { Patients diagnosed with colorectal cancer until } 2014 \\
(n=4,578)\end{array}$ \\
\hline
\end{tabular}

\begin{tabular}{|l|l|}
\hline & $\begin{array}{l}\text { Patients without tumor tissue were excluded } \\
(n=2,958)\end{array}$ \\
\hline \begin{tabular}{|l|l|} 
Patients with available colorectal cancer tissue \\
$(n=1,620)$
\end{tabular} \\
\hline $\begin{array}{l}\text { Patients without tumor LINE-1 methylation } \\
\text { data were excluded } \\
(n=264)\end{array}$ \\
\begin{tabular}{|l} 
Study population \\
Colorectal cancer patients with available tumor \\
molecular data ( $n=1,356)$ \\
age $<50$ (early-onset, $n=28)$ \\
age 50-54 (intermediate-onset, $n=66)$ \\
age 55-69 (n=614) \\
age $\geq 70(n=648)$
\end{tabular} \\
\hline
\end{tabular}

Figure 1. Flow chart of case selection in the Nurses' Health Study and the Health Professionals Followup Study. Abbreviations: HPFS, Health Professionals Follow-up Study; LINE-1, long interspersed nucleotide element-1; NHS, Nurses' Health Study.

The treatment of tissue lysates was conducted as previously reported $[17,24]$. Finally, we used the eluted DNA (80 $\mu \mathrm{L}$ volume) for pyrosequencing and MethyLight analysis $[17,24]$.

We performed the polymerase chain reaction (PCR) and pyrosequencing assay using the PyroMark kit (No. 978703, Qiagen, Valencia, CA, USA). This assay was conducted to amplify a region of the LINE-1 element (position 305 to 331 in accession No. X58075) which includes $4 \mathrm{CpG}$ sites (Figure 2). The PCR condition was as follows; (1) $95^{\circ} \mathrm{C}$ for $20 \mathrm{~s}\left(45\right.$ cycles); (2) $50{ }^{\circ} \mathrm{C}$ for $20 \mathrm{~s}$ and $72{ }^{\circ} \mathrm{C}$ for $20 \mathrm{~s}$; and (3) $72{ }^{\circ} \mathrm{C}$ for $5 \mathrm{~min}$. Using the Pyrosequencing Vacuum Prep Tool (Qiagen), we purified the biotinylated PCR product to make single-stranded nucleotides as a template in a pyrosequencing reaction. Then, we performed pyrosequencing reactions in the PSQ HS 96 System (Qiagen). The nucleotide dispensation order was as follows: 5'-ACT CAG TGT GTC AGT CAG TTA GTC TG-3'. We examined a single cytosine at a non-CpG site within PCR products to ensure successful 
bisulfite conversion of unmethylated cytosine based on evidence that the non-CpG cytosine in LINE-1 repetitive sequences was rarely methylated [25].

We calculated the percentage of the amount of $C$ nucleotides divided by the sum of the amounts of $\mathrm{C}$ and $\mathrm{T}$ nucleotides at each $\mathrm{CpG}$ site. We calculated the average of the relative amounts of $C$ nucleotides in the $4 \mathrm{CpG}$ sites in LINE-1. This average percentage value (a unitless number on a scale of 0 to 100) was used as the LINE-1 methylation level of each tumor. To avoid confusion with other \% numbers, we did not use "\%" in this measure of the LINE-1 methylation level. Figure 3 shows the validation procedure to assess the precision of bisulfite conversion and PCR-pyrosequencing. We performed PCRpyrosequencing seven times on each bisulfite-treated DNA and ensured a high precision of the LINE-1 methylation pyrosequencing assay [24]. We previously showed that DNA hypomethylation could be measured by using manual dissection without an LCM, and that the precision of measurement by using manual dissection was superior to cancer cells collected by LCM [24].

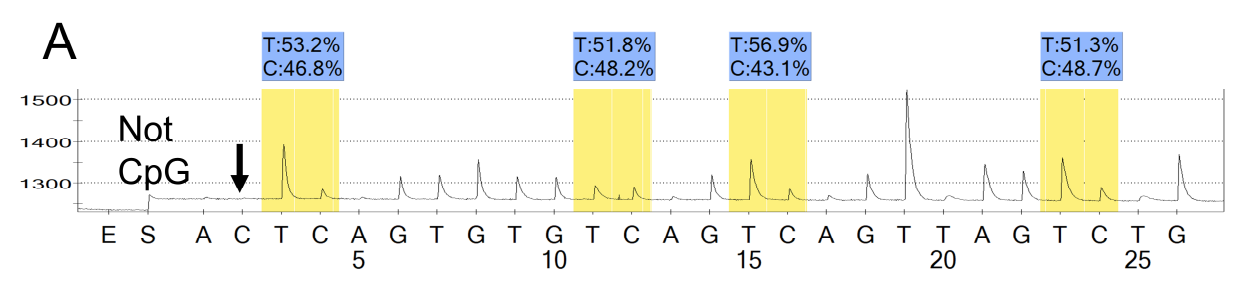

LINE-1 methylation level, 46.7

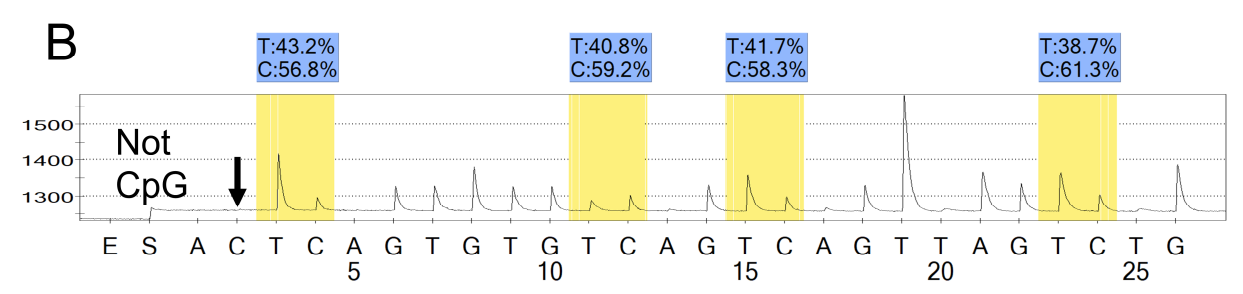

LINE-1 methylation level, 58.9

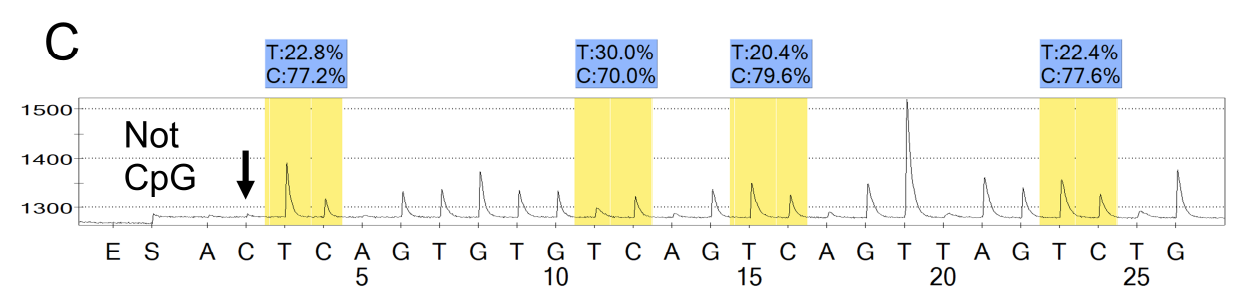

LINE-1 methylation level, 76.1

Figure 2. Pyrosequencing assay to measure LINE-1 methylation. (A) Tumor with LINE-1 hypomethylation (methylation level of 46.7). (B) Tumor with LINE-1 methylation level of 58.9. (C) Tumor with LINE-1 methylation level of 76.1. In panels A-C, the \% numbers (in blue shade) are proportions of T and $\mathrm{C}$ nucleotides at each CpG site after bisulfite conversion. The proportion of $\mathrm{C}$ nucleotides (\%) can be interpreted as the methylation level of each $\mathrm{CpG}$ site. The first, third, and fourth $\mathrm{CpG}$ sites follow mononucleotide $\mathrm{T}$ repeats, resulting in higher T peaks (in yellow shade) than the second $\mathrm{CpG}$ site. Accordingly, the proportion of $\mathrm{C}$ nucleotides (\%) has been adjusted. No residual $\mathrm{C}$ nucleotides at the non-CpG site are indicated by the arrows, providing evidence for a complete bisulfite conversion reaction. We used the average of the proportions of $\mathrm{C}$ nucleotides at the $4 \mathrm{CpG}$ sites as the LINE-1 methylation level (a scale of 0 to 100) of each tumor. To avoid confusion with other \% numbers, " $\%$ " is not used for this LINE-1 methylation level to keep consistency between the text, tables, and figures. Abbreviation: LINE-1, long interspersed nucleotide element-1. 


\subsection{Assessments of Other Tumor Characteristics}

DNA was extracted from archival FFPE blocks of normal and carcinomatous colorectal tissue. Methylation status of eight CIMP-specific promoters (CACNA1G, CDKN2A, CRABP1, IGF2, MLH1, NEUROG1, RUNX3, and SOCS1) was defined by the MethyLight assay using bisulphite-treated DNA, as described previously $[19,23,26]$ (following nomenclature recommendations for genes and products by an expert panel [27]). CIMP-high was defined as $\geq 6 / 8$ methylated markers using the 8-marker CIMP panel, CIMP-low as $1-5 / 8$ methylated promoters, and CIMP-negative as $0 / 8$ methylated promoters, according to previously established criteria [19,23]. Microsatellite instability (MSI) status was defined using PCR of 10 microsatellite markers (D2S123, D5S346, D17S250, BAT25, BAT26, BAT40, D18S55, D18S56, D18S67, and D18S487); MSI-high was defined as the presence of instability in $\geq 30 \%$ of the markers, as previously described [23,26]. PCR and pyrosequencing were performed for KRAS (codons 12, 13, 61, and 146), BRAF (codon 600), and PIK3CA (exons 9 and 20) [23,28].

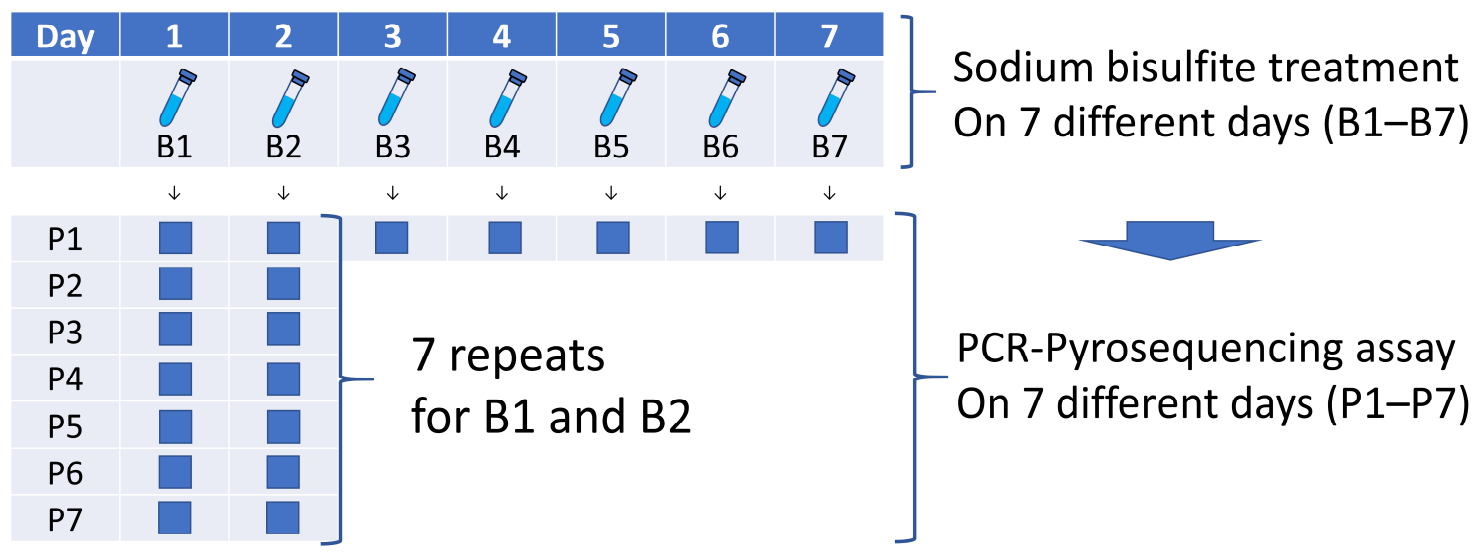

Figure 3. Validation procedure to assess precision of bisulfite conversion and PCR-pyrosequencing. Bisulfite conversion was performed on seven aliquots (B1 to B7) from each specimen. PCR-pyrosequencing was performed for seven bisulfite-treated specimens (B1 to B7) and was repeated seven times on two specimens (B1 and B2) on seven different days (P1 to P7). Abbreviation: PCR, polymerase chain reaction.

\subsection{Statistical Analyses}

All statistical analyses were performed using the SAS software (version 9.4, SAS Institute, Cary, NC, U.S.). All $p$ values were two-sided. We used the stringent two-sided $\alpha$ level of 0.005 , as recommended by a panel of expert statisticians [29]. Our primary hypothesis testing was an assessment of the association of age at diagnosis (age groups) with LINE-1 methylation level. All other assessments were secondary analyses. Spearman's correlation test was used to examine the association between four age groups and categorical data (or continuous values of LINE-1 methylation level). To control for other factors, multivariable-adjusted linear regression analyses were conducted with LINE-1 methylation level as an outcome variable. We initially included the following covariates: sex, body mass index ( $<30 \mathrm{vs.} \geq 30 \mathrm{~kg} / \mathrm{m}^{2}$ ), pack-years of smoking ( 0 vs. $1-39 \mathrm{vs.} \geq 40$ ), family history of colorectal cancer in any first-degree relative (present vs. absent), tumor location (proximal colon vs. distal colon vs. rectum), CIMP status (CIMP-negative/low vs. CIMP-high), MSI status (non-MSI-high vs. MSI-high), KRAS mutation (mutant vs. wild-type), BRAF mutation (mutant vs. wild-type), and PIK3CA mutation (mutant vs. wild-type). A backward elimination was conducted with a threshold $p$ value of 0.05 to select variables for the final model. Cases with missing data (body mass index $(0.4 \%)$, packyears of smoking $(4.4 \%)$, family history of colorectal cancer $(0.7 \%)$, tumor location $(0.5 \%)$, MSI status (2.8\%), CIMP status (4.9\%), KRAS mutation (6.4\%), BRAF mutation (2.2\%), and PIK3CA mutation $(8.7 \%)$ ) were imputed to the majority category of a given categorical 
covariate to limit the degrees of freedom of the models. Analyses using indicator variables for missing data in the variables in the final model yielded similar results (Table S1).

In survival analyses, cumulative survival probabilities were estimated with the KaplanMeier method, and a linear trend in survival probability across ordinal categories of LINE-1 methylation level was determined using the log-rank test for trend. Survival time was defined as the period from diagnosis of colorectal cancer to death or the end of follow-up, whichever came first. For the analyses of colorectal cancer-specific mortality, deaths due to other causes were censored. Multivariable Cox proportional hazard regression analyses were conducted for the colorectal cancer-specific survival according to the LINE-1 methylation level ( $\leq 45$ vs. $45-55$ vs. $55-65$ vs. $>65$ ). A $p$ value for trend was calculated using LINE-1 methylation level as a continuous variable with the same set of covariates. In the multivariable Cox regression model, we initially included the following covariates: age (continuous values), sex, body mass index $\left(<30 \mathrm{vs} . \geq 30 \mathrm{~kg} / \mathrm{m}^{2}\right)$, pack-years of smoking ( 0 vs. $1-39$ vs. $\geq 40$ ), family history of colorectal cancer in any first-degree relative (present vs. absent), tumor location (proximal colon vs. distal colon vs. rectum), tumor differentiation (well to moderate vs. poor), AJCC disease stage (I-II vs. III-IV), CIMP status (CIMP-negative/low vs. CIMP-high), MSI status (non-MSI-high vs. MSI-high), KRAS mutation (mutant vs. wild-type), BRAF mutation (mutant vs. wild-type), and PIK3CA mutation (mutant vs. wild-type). A backward elimination was conducted with a threshold $p$ of 0.05 to select variables for the final models. Cases with missing data (body mass index $(0.4 \%)$, pack-years of smoking $(4.4 \%)$, family history of colorectal cancer $(0.7 \%)$, tumor location $(0.5 \%)$, tumor differentiation ( $0.6 \%)$, AJCC disease stage $(9.1 \%)$, MSI status $(2.8 \%)$, CIMP status (4.9\%), KRAS mutation (6.4\%), BRAF mutation (2.2\%), and PIK3CA mutation $(8.7 \%))$ were imputed to the majority category of a given categorical covariate to limit the degrees of freedom of the models. The proportionality of the hazard assumption was assessed using a time-varying covariate, which is an interaction term of survival time and LINE-1 methylation level. The proportionality of the hazard assumption was satisfied for the analyses of cancer-specific survival $(p=0.34)$.

\subsection{Use of Standardized Official Symbols}

We use HUGO (Human Genome Organisation)-approved official symbols for genes and gene products, including BRAF, CACNA1G, CDKN2A, CRABP1, IGF2, KRAS, MLH1, NEUROG1, PIK3CA, RUNX3, and SOCS1, all of which are described at www.genenames. org accessed on 20 April 2021. The official symbols are italicized to differentiate from non-italicized colloquial names that are used along with the official symbols.

\section{Results}

In this study, we utilized a database of 1356 colorectal cancer cases with available tumor LINE-1 methylation data in the two prospective cohort studies, including 28 earlyonset cases (age <50) and 66 intermediate-onset cases (age 50-54). Tumor LINE-1 methylation levels (unitless values on a scale of 0 to 100; derived from percentage numbers) ranged from 23.1 to 93.8 (mean 63.6; standard deviation (SD) 10.1).

Table 1 summarizes the clinical, pathological, and molecular characteristics according to four age groups of $\geq 70,55-69,50-54$, and $<50$ years (Table S2 showing data using further categorization of $\geq 70,65-69,60-64,55-59,50-54$, and $<50$ years). In our primary hypothesis testing, younger age at diagnosis was associated with a lower tumor LINE-1 methylation level $(p<0.0001)$. The mean (with standard deviation (SD)) of the LINE-1 methylation level was 64.7 (SD 10.4) in age $\geq 70,62.8$ (SD 9.4) in age 55-69, 61.0 (SD 10.2) in age 50-54, and 58.9 (SD 12.0) in age $<50(p<0.0001)$ (Figure 4).

We categorized the LINE-1 methylation level into four ordinal subtypes using arbitrary cut points of 45, 55, and 65 (Table 1). A tumor LINE- 1 methylation level of $\leq 55$ was uncommon in colorectal cancer patients aged $>70$ and those aged 55-69, but more common in those aged 50-54 and most common in those aged $<50(p=0.0005)$. Conversely, a tumor 
LINE-1 methylation level of $>65$ was most common in patients aged $>70$, followed by those aged 55-69 and those aged 50-54, and least common in those aged $<50$.

To adjust for other factors, we conducted multivariable-adjusted linear regression analysis that could assess the association of age at diagnosis with the tumor LINE-1 methylation level as a continuous variable (Table 2). As shown in Table 1, early-onset colorectal cancers in this dataset were associated with some clinical and tumor molecular features, such as female sex, rectal location, non-MSI-high status, and CIMP-negative status. There was evidence for a certain degree of confounding, manifested as a difference in the $\beta$ coefficients (i.e., difference in the mean LINE-1 methylation level by a given variable) between the unadjusted and adjusted models. However, even after the adjustment in the multivariable model, there was a highly significant association of four age groups with the LINE-1 methylation level ( $P_{\text {trend }}<0.0001$ across the age groups). Compared to patients aged $\geq 70$, the multivariable-adjusted $\beta$ coefficient for the continuous LINE-1 methylation level was -1.38 (95\% confidence interval (CI), -2.47 to -0.30 ) for age 55-69, $-2.82(95 \% \mathrm{CI},-5.29$ to -0.34$)$ for age $50-54$, and -4.54 (95\% CI, -8.24 to -0.85 ) for age $<50\left(P_{\text {trend }}=0.0003\right.$ across the age groups) (Table 2$)$.

Table 1. Clinical, pathological, and molecular characteristics of colorectal cancer cases according to age at diagnosis.

\begin{tabular}{|c|c|c|c|c|c|c|}
\hline \multirow[b]{2}{*}{ Characteristics $^{a}$} & \multirow{2}{*}{$\begin{array}{l}\text { Total No. } \\
(n=1356)\end{array}$} & \multicolumn{4}{|c|}{ Age at Diagnosis } & \multirow[b]{2}{*}{$p$ Value $^{b}$} \\
\hline & & $\begin{array}{c}<50 \\
(n=28)\end{array}$ & $\begin{array}{c}50-54 \\
(n=66)\end{array}$ & $\begin{array}{c}55-69 \\
(n=614)\end{array}$ & $\begin{array}{c}\geq 70 \\
(n=648)\end{array}$ & \\
\hline Sex & & & & & & $<0.0001$ \\
\hline Female (NHS) & $742(55 \%)$ & $21(75 \%)$ & $46(70 \%)$ & $378(62 \%)$ & $297(46 \%)$ & \\
\hline Male (HPFS) & $614(45 \%)$ & $7(25 \%)$ & $20(30 \%)$ & $236(38 \%)$ & $351(54 \%)$ & \\
\hline Body mass index & & & & & & 0.42 \\
\hline$<30 \mathrm{~kg} / \mathrm{m}^{2}$ & $1099(81 \%)$ & $23(82 \%)$ & $54(82 \%)$ & $491(80 \%)$ & $531(82 \%)$ & \\
\hline$\geq 30 \mathrm{~kg} / \mathrm{m}^{2}$ & $251(19 \%)$ & $5(18 \%)$ & $12(18 \%)$ & $121(20 \%)$ & $113(18 \%)$ & \\
\hline Pack-years of smoking & & & & & & 0.020 \\
\hline 0 & $531(41 \%)$ & $14(50 \%)$ & $40(63 \%)$ & $233(39 \%)$ & $244(40 \%)$ & \\
\hline $1-39$ & $547(42 \%)$ & $13(46 \%)$ & $24(37 \%)$ & $257(43 \%)$ & $253(41 \%)$ & \\
\hline$\geq 40$ & $219(17 \%)$ & $1(3.6 \%)$ & 0 & $103(17 \%)$ & $115(19 \%)$ & \\
\hline $\begin{array}{l}\text { Family history of colorectal } \\
\text { cancer }\end{array}$ & & & & & & 0.75 \\
\hline Absent & $1077(80 \%)$ & $22(79 \%)$ & $51(80 \%)$ & $492(81 \%)$ & $512(80 \%)$ & \\
\hline Present & $270(20 \%)$ & $6(21 \%)$ & $13(20 \%)$ & $119(19 \%)$ & $132(20 \%)$ & \\
\hline Tumor location & & & & & & $<0.0001$ \\
\hline Proximal colon & $641(48 \%)$ & $6(21 \%)$ & $26(39 \%)$ & $258(42 \%)$ & $351(54 \%)$ & \\
\hline Distal colon & $410(30 \%)$ & $14(50 \%)$ & $26(39 \%)$ & $204(33 \%)$ & $166(26 \%)$ & \\
\hline Rectum & $298(22 \%)$ & $8(29 \%)$ & $14(22 \%)$ & $150(25 \%)$ & $126(20 \%)$ & \\
\hline pT stage & & & & & & 0.76 \\
\hline pT1 & $143(12 \%)$ & $3(11 \%)$ & $4(6.4 \%)$ & $76(13 \%)$ & $60(10 \%)$ & \\
\hline pT2 & $259(21 \%)$ & $8(30 \%)$ & $13(21 \%)$ & $105(19 \%)$ & $133(23 \%)$ & \\
\hline pT3 & $773(62 \%)$ & $13(48 \%)$ & $41(65 \%)$ & $357(63 \%)$ & $362(62 \%)$ & \\
\hline pT4 & $67(5.4 \%)$ & $3(11 \%)$ & $5(8.0 \%)$ & $28(5.0 \%)$ & $31(5.3 \%)$ & \\
\hline pN stage & & & & & & 0.048 \\
\hline pN0 (0) & $758(63 \%)$ & $16(62 \%)$ & $25(45 \%)$ & $346(63 \%)$ & $371(65 \%)$ & \\
\hline $\mathrm{pN} 1(1-3)$ & $277(23 \%)$ & $4(15 \%)$ & $18(33 \%)$ & $127(23 \%)$ & $128(23 \%)$ & \\
\hline $\mathrm{pN} 2(\geq 4)$ & $166(14 \%)$ & $6(23 \%)$ & $12(22 \%)$ & $77(14 \%)$ & $71(12 \%)$ & \\
\hline AJCC disease stage & & & & & & 0.026 \\
\hline I & $315(26 \%)$ & $9(32 \%)$ & $9(15 \%)$ & $141(25 \%)$ & $156(27 \%)$ & \\
\hline II & $394(32 \%)$ & $7(25 \%)$ & $13(22 \%)$ & $180(32 \%)$ & $194(34 \%)$ & \\
\hline III & $351(28 \%)$ & $9(32 \%)$ & $26(43 \%)$ & $162(29 \%)$ & $154(26 \%)$ & \\
\hline IV & $172(14 \%)$ & $3(11 \%)$ & $12(20 \%)$ & $83(14 \%)$ & $74(13 \%)$ & \\
\hline Tumor differentiation & & & & & & 0.59 \\
\hline Well to moderate & $1212(90 \%)$ & $26(93 \%)$ & $59(89 \%)$ & $551(90 \%)$ & $576(89 \%)$ & \\
\hline Poor & $136(10 \%)$ & $2(7.0 \%)$ & $7(11 \%)$ & $59(9.7 \%)$ & $68(11 \%)$ & \\
\hline \multirow{2}{*}{$\begin{array}{l}\text { LINE-1 methylation level } \\
\text { (0 to } 100 \text { (percent) scale) } \\
\text { mean } \pm \text { SD }\end{array}$} & & & & & & $<0.0001$ \\
\hline & $63.6 \pm 10.1$ & $58.9 \pm 12.0$ & $61.0 \pm 10.2$ & $62.8 \pm 9.4$ & $64.7 \pm 10.4$ & \\
\hline
\end{tabular}


Table 1. Cont.

\begin{tabular}{|c|c|c|c|c|c|c|}
\hline \multirow[b]{2}{*}{ Characteristics $^{a}$} & \multirow{2}{*}{$\begin{array}{l}\text { Total No. } \\
(n=1356)\end{array}$} & \multicolumn{4}{|c|}{ Age at Diagnosis } & \multirow[b]{2}{*}{$p$ Value $^{\mathrm{b}}$} \\
\hline & & $\begin{array}{c}<50 \\
(n=28)\end{array}$ & $\begin{array}{c}50-54 \\
(n=66)\end{array}$ & $\begin{array}{c}55-69 \\
(n=614)\end{array}$ & $\begin{aligned} & \geq 70 \\
&(n=648)\end{aligned}$ & \\
\hline LINE-1 methylation level & & & & & & 0.0005 \\
\hline$\leq 45$ & $47(3.5 \%)$ & $5(18 \%)$ & $2(3.0 \%)$ & $21(3.4 \%)$ & $19(2.9 \%)$ & \\
\hline $45-55$ & $206(15 \%)$ & $4(14 \%)$ & $15(23 \%)$ & $98(16 \%)$ & $89(14 \%)$ & \\
\hline $55-65$ & $499(37 \%)$ & $10(36 \%)$ & $23(35 \%)$ & $245(40 \%)$ & $221(34 \%)$ & \\
\hline$>65$ & $604(45 \%)$ & $9(32 \%)$ & $26(39 \%)$ & $250(41 \%)$ & $319(49 \%)$ & \\
\hline MSI status & & & & & & $<0.0001$ \\
\hline Non-MSI-high & $1101(84 \%)$ & $26(100 \%)$ & $56(89 \%)$ & $520(87 \%)$ & $499(79 \%)$ & \\
\hline MSI-high & $217(16 \%)$ & $0(0 \%)$ & $7(11 \%)$ & $80(13 \%)$ & $130(21 \%)$ & \\
\hline CIMP status & & & & & & $<0.0001$ \\
\hline Negative & $553(43 \%)$ & $17(61 \%)$ & $37(58 \%)$ & $270(46 \%)$ & $229(38 \%)$ & \\
\hline Low & $507(39 \%)$ & $10(36 \%)$ & $23(36 \%)$ & $240(40 \%)$ & $234(39 \%)$ & \\
\hline High & $230(18 \%)$ & $1(3.6 \%)$ & $4(6.3 \%)$ & $83(14 \%)$ & $142(23 \%)$ & \\
\hline$K R A S$ mutation & & & & & & 0.22 \\
\hline Wild-type & $737(58 \%)$ & $16(62 \%)$ & $41(66 \%)$ & $346(59 \%)$ & $334(57 \%)$ & \\
\hline Mutant & $532(42 \%)$ & $10(38 \%)$ & $21(34 \%)$ & $245(41 \%)$ & $256(43 \%)$ & \\
\hline$B R A F$ mutation & & & & & & 0.024 \\
\hline Wild-type & $1124(85 \%)$ & $24(92 \%)$ & $56(89 \%)$ & $522(86 \%)$ & $522(83 \%)$ & \\
\hline Mutant & $202(15 \%)$ & $2(7.7 \%)$ & $7(11 \%)$ & $83(14 \%)$ & $110(17 \%)$ & \\
\hline PIK3CA mutation & & & & & & 0.63 \\
\hline Wild-type & $1039(84 \%)$ & $22(85 \%)$ & $49(86 \%)$ & $463(84 \%)$ & $505(83 \%)$ & \\
\hline Mutant & $199(16 \%)$ & $4(15 \%)$ & $8(14 \%)$ & $87(16 \%)$ & $100(17 \%)$ & \\
\hline
\end{tabular}

a Percentage indicates the proportion of patients with a specific clinical, pathological, or molecular characteristic among all patients or in the strata of age at diagnosis. ${ }^{b}$ Spearman's correlation test was used to examine the association between four age groups ( $\geq 70,55-69$, 50-54, and <50) and categorical data (or continuous value of LINE-1 methylation level). Abbreviations: AJCC, American Joint Committee on Cancer; CIMP, CpG island methylator phenotype; HPFS, Health Professionals Follow-up Study; LINE-1, long interspersed nucleotide element-1; MSI, microsatellite instability; NHS, Nurses' Health Study; SD, standard deviation.

In the survival analyses, using a dataset of 1352 cases with available survival data, we examined the prognostic impact of the LINE-1 methylation level. During the median follow-up time of 9.8 years (interquartile range, 3.8 to 16.2 years), 945 all-cause deaths, including 413 colorectal cancer-specific deaths, were observed. Kaplan-Meier analysis showed that LINE-1 hypomethylation was associated with higher colorectal cancer-specific mortality ( $\log$-rank $p=0.0001$ ) (Figure 5 and Table 3). Multivariable Cox regression models indicated that LINE-1 hypomethylation was associated with higher colorectal cancerspecific mortality independent of tumor molecular features and patient characteristics. Multivariable-adjusted hazard ratios (95\% CI) for LINE-1 methylation levels of $\leq 45,45-55$, and 55-65 (vs. >65) were 2.33 (1.49 to 3.64), 1.39 (1.05 to 1.85), and 1.29 (1.02 to 1.63), respectively $\left(P_{\text {trend }}=0.0005\right)$ (Table 4$)$. 


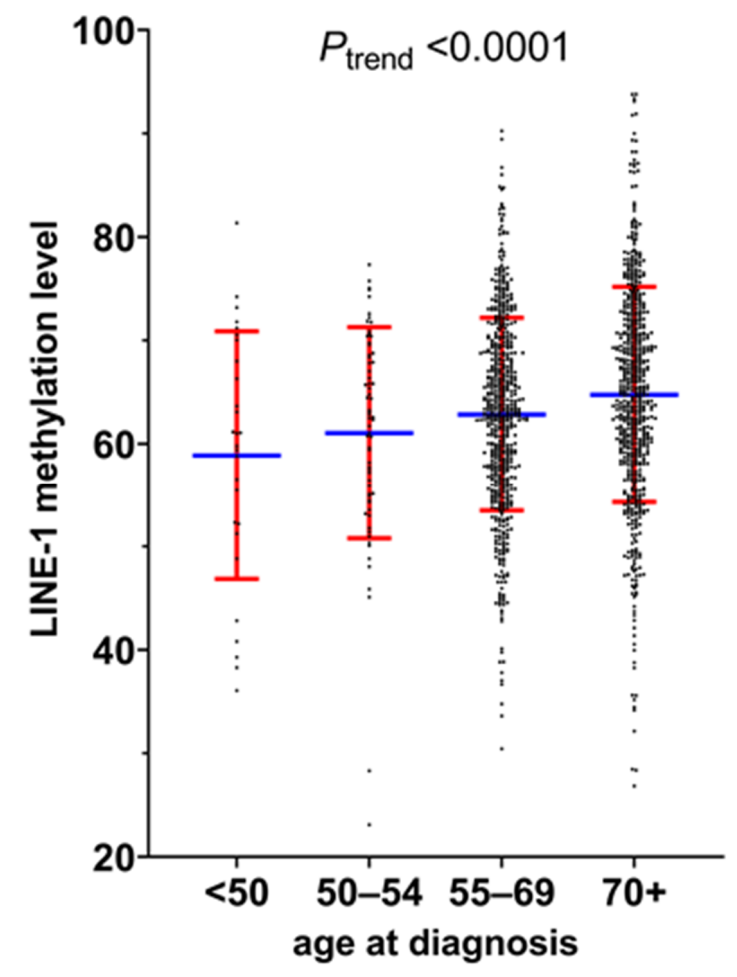

Figure 4. Distribution of tumor LINE-1 methylation levels in different age groups. In the scatter dot plot, the blue horizontal bar marks the mean and the red horizontal bar indicates the standard deviation of tumor LINE-1 methylation levels in each age group. A significant difference was observed between four age groups $(\geq 70,55-69,50-54$, and $<50)\left(P_{\text {trend }}<0.0001\right)$. Abbreviation: LINE-1, long interspersed nucleotide element-1.

Table 2. Linear regression analysis to predict LINE-1 methylation level (outcome) by four age groups (predictor).

\begin{tabular}{|c|c|c|c|c|}
\hline \multirow{2}{*}{$\begin{array}{l}\text { Variables in the Final } \\
\text { Model }\end{array}$} & \multicolumn{4}{|c|}{$\begin{array}{c}\beta \text { Coefficient }^{\text {a }} \\
\text { (Change in Mean LINE-1 Methylation Levels by a Given Variable) }\end{array}$} \\
\hline & $\begin{array}{l}\text { Univariable (Unadjusted) } \\
\qquad(95 \% \mathrm{CI})\end{array}$ & $p$ Value & $\begin{array}{l}\text { Multivariable-Adjusted }{ }^{b} \\
\qquad(95 \% \text { CI })\end{array}$ & $p$ Value \\
\hline Age at diagnosis & & $<0.0001^{\mathrm{c}}$ & & $0.0003^{c}$ \\
\hline$<50$ & $-5.87(-9.65$ to -2.09$)$ & & $-4.54(-8.24$ to -0.85$)$ & \\
\hline $50-54$ & $-3.70(-6.23$ to -1.17$)$ & & $-2.82(-5.29$ to -0.34$)$ & \\
\hline $55-69$ & $-1.90(-3.00$ to -0.80$)$ & & $-1.38(-2.47$ to -0.30$)$ & \\
\hline$\geq 70$ & Referent & & Referent & \\
\hline $\begin{array}{l}\text { Family history of colorectal } \\
\text { cancer }\end{array}$ & & 0.13 & & 0.029 \\
\hline Absent & Referent & & Referent & \\
\hline Present & $-1.03(-2.37$ to 0.31$)$ & & $-1.45(-2.75$ to -0.15$)$ & \\
\hline MSI status & & $<0.0001$ & & 0.002 \\
\hline Non-MSI-high & Referent & & Referent & \\
\hline MSI-high & $5.73(4.30$ to 7.16$)$ & & $2.99(1.14$ to 4.84$)$ & \\
\hline CIMP status & & $<0.0001$ & & $<0.0001$ \\
\hline Negative/low & Referent & & Referent & \\
\hline High & $6.00(4.61$ to 7.39$)$ & & $3.86(2.05$ to 5.67$)$ & \\
\hline
\end{tabular}

${ }^{a}$ The $\beta$ coefficient represents a change (increase or decrease) in mean LINE- 1 methylation level by a given variable. ${ }^{b}$ The multivariable linear regression model initially included age, sex, body mass index, pack-years of smoking, family history of colorectal cancer in any first-degree relative, tumor location, MSI status, CIMP status, and KRAS, BRAF, and PIK3CA mutations. A backward elimination with a threshold $p$ of 0.05 was performed to select variables in the final model. The variables listed in Table 2 remained in the final model. ${ }^{c} P_{\text {trend }}$ was calculated by the linear trend across the ordinal age variable $(\geq 70,55-69,50-54$, and $<50)$ in the linear regression model. Abbreviations: CI, confidential interval; CIMP, CpG island methylator phenotype; LINE-1, long interspersed nucleotide element-1; MSI, microsatellite instability. 

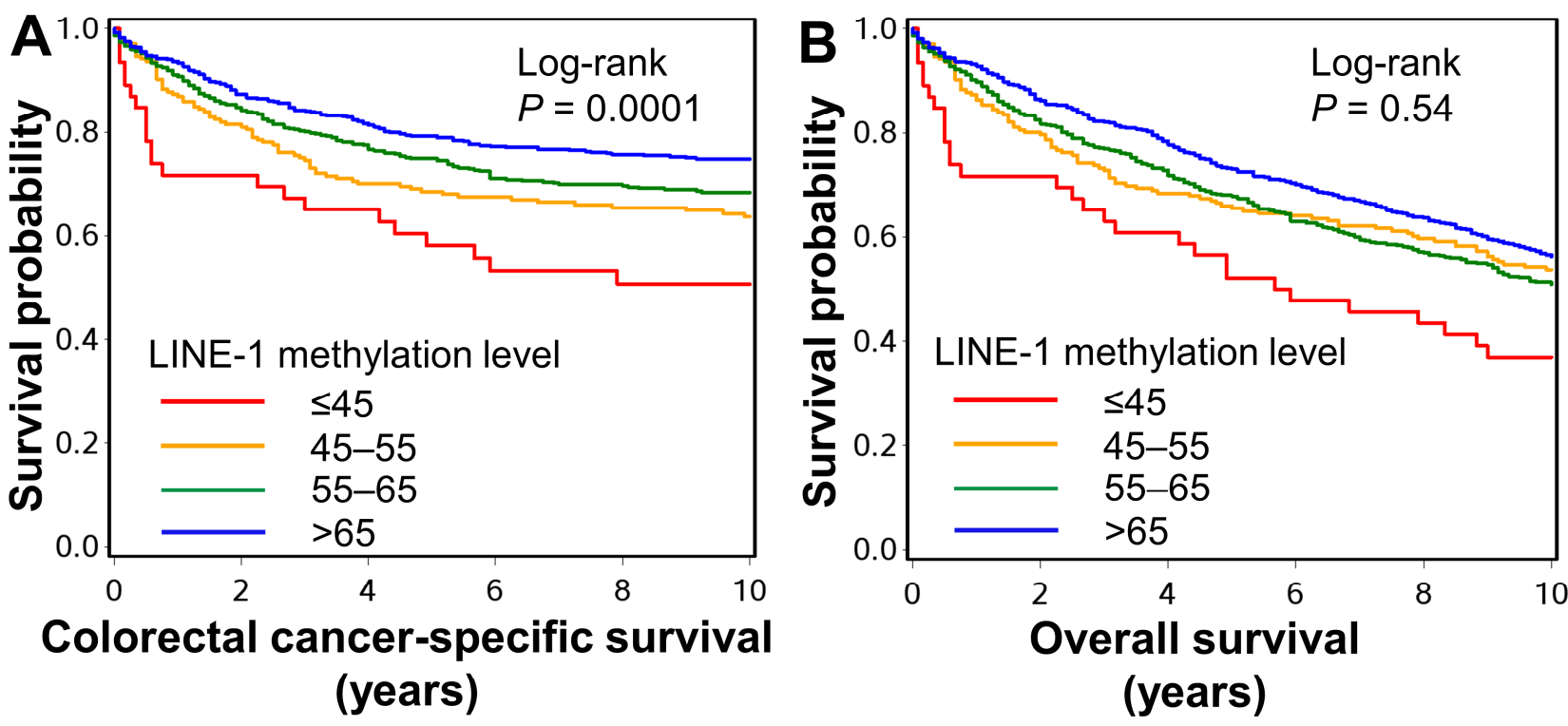

Figure 5. Kaplan-Meier survival curves of colorectal cancer-specific survival (A) and overall survival (B) according to the LINE-1 methylation level. The $p$ values were calculated using the log-rank test for trend (two-sided).

Table 3. Number at risk of death during follow-up of patients according to tumor LINE-1 methylation levels

\begin{tabular}{ccccccc}
\hline \multirow{2}{*}{ LINE-1 Methylation Level } & \multicolumn{7}{c}{ Years } \\
\cline { 2 - 7 } & $\mathbf{0}$ & $\mathbf{2}$ & $\mathbf{4}$ & $\mathbf{6}$ & $\mathbf{8}$ & $\mathbf{1 0}$ \\
\hline$\leq 45$ & 46 & 33 & 28 & 22 & 20 & 17 \\
$45-55$ & 206 & 164 & 141 & 132 & 122 & 108 \\
$55-65$ & 498 & 407 & 358 & 314 & 279 & 242 \\
$>65$ & 602 & 519 & 468 & 420 & 365 & 296 \\
\hline
\end{tabular}

This table shows the number of patients who remained alive and at risk of death at each time point after the diagnosis of colorectal cancer.

Table 4. Survival of colorectal cancer patients according to tumor LINE-1 methylation levels.

\begin{tabular}{|c|c|c|c|c|c|c|c|}
\hline \multirow[b]{2}{*}{$\begin{array}{c}\text { LINE-1 } \\
\text { Methylation Level }\end{array}$} & \multirow[b]{2}{*}{$\begin{array}{l}\text { No. of } \\
\text { Cases }\end{array}$} & \multicolumn{3}{|c|}{ Colorectal Cancer-Specific Survival } & \multicolumn{3}{|c|}{ Overall Survival } \\
\hline & & $\begin{array}{l}\text { No. of } \\
\text { Events }\end{array}$ & $\begin{array}{l}\text { Univariable } \\
\text { HR }(95 \% \text { CI) }\end{array}$ & $\begin{array}{l}\text { Multivariable } \\
\text { HR }(95 \% \text { CI) }\end{array}$ & $\begin{array}{l}\text { No. of } \\
\text { Events }\end{array}$ & $\begin{array}{l}\text { Univariable } \\
\text { HR }(95 \% \text { CI) }\end{array}$ & $\begin{array}{l}\text { Multivariable } \\
\text { HR (95\% CI) }\end{array}$ \\
\hline$\leq 45$ & 46 & 22 & 2.26 (1.41 to 3.62$)$ & $2.33(1.49$ to 3.64$)$ & 34 & $1.09(0.70$ to 1.69$)$ & $1.63(1.07$ to 2.49$)$ \\
\hline $45-55$ & 206 & 80 & $1.60(1.22$ to 1.16$)$ & 1.39 (1.05 to 1.85$)$ & 150 & $1.01(0.84$ to 1.23$)$ & $1.10(0.90$ to 1.33$)$ \\
\hline $55-65$ & 498 & 157 & $1.28(1.03$ to 1.60$)$ & $1.29(1.02$ to 1.63$)$ & 365 & 1.11 (0.96 to 1.27$)$ & $1.21(1.05$ to 1.40$)$ \\
\hline$>65$ & 602 & 154 & Referent & Referent & 396 & Referent & Referent \\
\hline$P_{\text {trend }} \mathrm{b}$ & & & 0.0002 & 0.0005 & & 0.92 & 0.020 \\
\hline
\end{tabular}

a The multivariable Cox regression model initially included age, sex, body mass index, pack-years of smoking, family history of colorectal cancer in any first-degree relative, tumor location, tumor differentiation, disease stage, microsatellite instability, CpG island methylator phenotype, and KRAS, BRAF, and PIK3CA mutations. A backward elimination with a threshold $p$ of 0.05 was used to select variables for the final models. ${ }^{\mathrm{b}} P_{\text {trend }}$ was calculated using LINE-1 methylation level as a continuous variable with the same set of covariates. Abbreviations: $\mathrm{CI}$, confidence interval; HR, hazard ratio; LINE-1, long interspersed nucleotide element-1.

\section{Discussion}

We conducted this study to assess the association of age at colorectal cancer diagnosis with tumor LINE-1 hypomethylation, with a special focus on early-onset (age $<50)$ and intermediate-onset (age 50-54) cases. We leveraged the molecular pathological epidemiology database within the two prospective cohort studies that provided detailed information of clinicopathological features and tumor molecular profiles. Our findings of the associa- 
tion between early age of colorectal cancer diagnosis and tumor LINE-1 hypomethylation provide evidence for a greater pathogenic role of global DNA hypomethylation in colorectal cancers arising in younger age. Although the possible link between early-onset colorectal cancer and tumor LINE-1 hypomethylation has been reported [16], it has been unclear whether the link is independent of factors such as MSI status and CIMP. Furthermore, an open question is whether colorectal cancers diagnosed at age 50-54 (i.e., not early-onset cancer in the common definition, hence herein referred to as "intermediate-onset" cancer) have similar tumor characteristics to early-onset patients (or older patients). While replication is needed, our results suggest that intermediate-onset colorectal cancer patients (age 50-54) may exhibit tumor LINE-1 hypomethylation less commonly than early-onset cancer patients (age $<50$ ) but more commonly than older patients. These findings do not suggest a sharp biological dichotomy of early-onset vs. later-onset colorectal cancer (with age 50 as a cut point), but rather support an "age continuum" model, which has recently been proposed [7].

The incidence of early-onset colorectal cancer has been increasing around the world [30]. Between 2000 and 2017, the age-adjusted annual incidence of colorectal cancer increased from 5.9 to 8.4 cases per 100,000 persons in the USA [31]. Accumulating evidence indicates that, compared to later-onset colorectal cancers (usually used for colorectal cancers diagnosed at age 50 or above), early-onset colorectal cancers are associated with rectal location, advanced stage at diagnosis, poor tumor differentiation, and signet ring cell histology [9,32-36]. Previous studies reported a relatively high prevalence of specific germline genetic features in young colorectal cancer patients [8,37,38]. Evidence indicates that the molecular characteristics of early-onset and later-onset colorectal cancers might differ $[9,32-35,39]$. Although certain early-life exposures, such as obesity, might be associated with early-onset colorectal cancer [11], the precise reason behind the increase in the incidence of early-onset colorectal cancer remains unclear, in part because most existing epidemiological studies lack precise early-life information [7]. A growing body of epidemiological evidence suggests that potential risk factors associated with early-onset colorectal cancer include male sex [40], family history of colorectal cancer [40,41], obesity [11,40], diet such as processed meat [40,41], Black and Asian ethnicities [40], and high intake of alcohol [41].

The genetic background of human populations is largely static over the short term. It is conceivable that environmental factors and their influence on epigenetics may play a role in the rise of early-onset colorectal cancer [7]. Evidence suggests that endogenous and exogenous exposures may act on cellular epigenetic modulators that regulate gene expression $[12,42]$. Hence, epigenetics is considered to serve as a bridge between the environment and phenotypes $[7,14]$. Hence, there is importance in investigating the association between age of onset and epigenetic alterations in cancer $[16,17]$. Aberrant epigenetic processes, which may be promoted by diet, lifestyle, and environmental exposures throughout the life course, likely underlie the increasing incidence of various cancer types, which were observed more commonly in old individuals in young adults.

DNA methylation is an essential epigenetic process that modulates gene expression. Global DNA hypomethylation is likely a manifestation of widespread epigenomic instability that plays a role in carcinogenesis [15]. A recent study suggested that LINE-1 retrotransposition (which may be caused by LINE-1 hypomethylation) may be a major mechanism that causes somatic structural variation in various tumor types including colorectal cancer [43]. Previous studies have reported that tumor LINE-1 hypomethylation, which has been correlated with global DNA hypomethylation [44,45], is associated with younger age of onset [16,17], higher T stage [46], and shorter survival [16,19,20,46-50] in colorectal cancer. The tumor LINE-1 methylation level in metastatic tumors appeared to be lower than that in primary tumors in colorectal cancer [51]. We found that LINE-1 hypomethylation was independently associated with non-MSI-high status and CIMPnegative/low status. The CIMP-high status is a major phenotype involved in colorectal cancer development, where $\mathrm{CPG}$ island methylation is a mechanism of silencing gene 
expression [52,53]. We have previously reported that CIMP-high tumors are associated with proximal colon location, female sex, poor differentiation, MSI-high status, and BRAF mutations [54]. To our knowledge, the current study is the largest to evaluate tumor LINE-1 methylation in different age groups, including early-onset and intermediate-onset colorectal cancers.

In this study, we examined whether the prevalence of LINE-1 hypomethylation abruptly varied at age 50 or gradually changed according to age. Although age 50 is usually defined as the cut point for early-onset vs. later-onset colorectal cancer, our current study provides no evidence that the molecular characteristics abruptly change at the age of 50. Rather, our data provide support for the "age continuum" model [7], with regard to tumor LINE-1 hypomethylation, which reflects global DNA hypomethylation. This age continuum model in colorectal cancer has important biological and clinical implications. It is possible that the recommended starting age of 50 for colorectal cancer screening in the past provided the ready rationale of the dichotomy model (with the cut point at age 50) in colorectal cancer research. The past screening practice might have influenced the incidence of colorectal cancer around age 50 in complex ways. Recently, the American Cancer Society [55], the U.S. Preventive Services Task Force, and the American College of Gastroenterology [56] recommended starting screening at the age of 45 instead of 50; therefore, monitoring how colorectal cancer incidence will change accordingly is an important element of early-onset colorectal cancer research moving forward. Our current study highlights the importance of considering the plausible "age continuum" model in research on cancers in not only the colorectum but also many other body sites.

Our study has limitations. First, the sample size of patients with early-onset colorectal cancer analyzed in this study was limited. Nonetheless, a moderate number of intermediateonset cases and a large number of older patients in the molecular pathological epidemiology database enabled us to detect the statistically significant trend across the ordinal age groups of colorectal cancer. Second, there existed unmeasured and/or residual confounding in this observational study. However, we attempted to adjust for a number of factors such as body mass index, cigarette smoking, colorectal cancer family history, tumor location, CIMP, and MSI status in our multivariable-adjusted linear regression analysis. Third, most of the subjects in this study were non-Hispanic Whites. Therefore, a replication of findings in other populations is needed. Fourth, the bisulfite sequencing could not differentiate 5-methylcytosine from 5-hydroxymethylcytosine. Therefore, there was a possibility that some of the methylated $\mathrm{CpG}$ sites identified in the study might be due to 5-hydroxymethylcytosines.

The primary strength of this study was the utilization of the molecular pathological epidemiology approach [57-63], together with a large database of colorectal carcinoma cases, which integrated epidemiological, clinical, pathological, and tumor molecular features. This comprehensive database enabled us to conduct multivariable analysis adjusting for an extensive group of covariates. Moreover, the study subjects of incident colorectal cancer cases in the two prospective cohort studies were derived from over 100 hospitals throughout the U.S., which increases the generalizability of our findings, compared to studies based on a limited number of hospitals. In addition, our LINE-1 methylation assay on archival colorectal tumor tissue was extensively validated with well-documented robust performance characteristics including high precision [64].

\section{Conclusions}

This study indicates that younger age of colorectal cancer diagnosis is associated with lower LINE-1 methylation levels, potentially reflecting a greater pathogenic role of global DNA hypomethylation in colorectal cancer arising in younger adults. These findings support the "age continuum" model (as opposed to the common age dichotomy model at 50 years) in terms of tumor LINE-1 hypomethylation. The proposed "age continuum" model has substantial implications in research on cancers in the colorectum as well as other body sites. 
Supplementary Materials: The following available online at https:/ /www.mdpi.com/article/10.3 390/cancers13092016/s1, Table S1: LINE-1 methylation level of colorectal cancer cases according to age at diagnosis, Table S2: Linear regression analysis to predict LINE-1 methylation level (outcome) by four age groups (predictor) using indicator variables for missing data.

Author Contributions: Conceptualization, S.O.; methodology, S.O.; software, N.A., T.U., R.Z., and S.O.; validation, N.A., M.Z., and T.U.; formal analysis, N.A., M.Z., T.U., R.Z., J.P.V., and S.O.; investigation, N.A., M.Z., T.U., and S.O.; resources, S.O.; data curation, N.A., T.U., and S.O.; writingoriginal draft preparation, N.A., M.Z., T.U., R.Z., J.P.V., and S.O.; writing-review and editing, N.A., M.Z., T.U., R.Z., M.C.L., K.F., J.K., K.H., K.A., T.S.T., X.Z., E.L.G., K.W., M.S., A.T.C., Y.C., J.A.M., K.N., M.G., J.P.V., J.A.N., and S.O.; visualization, N.A., T.U., and S.O.; supervision, J.P.V. and S.O.; project administration, S.O.; funding acquisition, X.Z., E.L.G., K.W., M.S., A.T.C., Y.C., J.A.M., K.N., M.G., J.A.N., and S.O. The first four authors contributed equally as co-first authors. The last four authors contributed equally as co-last authors. All authors have read and agreed to the published version of the manuscript.

Funding: This work was supported by U.S. National Institutes of Health (NIH) grants (P01 CA87969; UM1 CA186107; P01 CA55075; UM1 CA167552; U01 CA167552; R35 CA253185 to A.T.C.; R37 CA246175 to Y.C.; R35 CA197735 to S.O.; R01 CA151993 to S.O.); by a Stand Up to Cancer Colorectal Cancer Dream Team Translational Research Grant (SU2C-AACR-DT22-17 to M.G.), administered by the American Association for Cancer Research, a scientific partner of SU2C; and by grants from the Project P Fund, The Friends of the Dana-Farber Cancer Institute, Bennett Family Fund, and the Entertainment Industry Foundation through National Colorectal Cancer Research Alliance and SU2C. X.Z. was supported by the American Cancer Society Research Scholar Grant (RSG NEC-130476). R.Z. was supported by a fellowship grant from Huazhong University of Science and Technology. K.F. was supported by fellowship grants from the Uehara Memorial Foundation and Grant of The Clinical Research Promotion Foundation (2018). K.A. and T.U. were supported by a grant from the Overseas Research Fellowship (201860083 to K.A.; 201960541 to T.U.) from the Japan Society for the Promotion of Science. K.H. was supported by fellowship grants from the Uehara Memorial Foundation and the Mitsukoshi Health and Welfare Foundation. A.T.C. is a Stuart and Suzanne Steele MGH Research Scholar. M.G. is supported by an ASCO Conquer Cancer Foundation Career Development Award. The content is solely the responsibility of the authors and does not necessarily represent the official views of NIH. The funders had no role in the study design, data collection and analysis, decision to publish, or preparation of the manuscript.

Institutional Review Board Statement: The study was conducted according to the guidelines of the Declaration of Helsinki and approved by the Institutional Review Board of Brigham and Women's Hospital and Harvard T.H. Chan School of Public Health (2001P001945) on 20 October 2020.

Informed Consent Statement: Informed consent was obtained from all subjects involved in the study.

Data Availability Statement: The datasets generated and/or analyzed during the current study are not publicly available. Further information including the procedures to obtain and access data from the Nurses' Health Studies and the Health Professionals Follow-up Study is described at https:/ / www.nurseshealthstudy.org/researchers/ accessed on 20 April 2021 and https:/ / sites.sph. harvard.edu/hpfs/for-collaborators/ accessed on 20 April 2021.

Acknowledgments: We would like to thank the participants and staff of the Nurses' Health Study and the Health Professionals Follow-up Study for their valuable contributions as well as the following state cancer registries for their help: A.L., A.Z., A.R., C.A., C.O., C.T., D.E., F.L., G.A., I.D., I.L., I.N., I.A., K.Y., L.A., M.E., M.D., M.A., M.I., N.E., N.H., N.J., N.Y., N.C., N.D., O.H., O.K., O.R., P.A., R.I., S.C., T.N., T.X., V.A., W.A., W.Y. The authors assume full responsibility for analyses and interpretation of these data.

Conflicts of Interest: J.A.M. has received institutional research funding from Boston Biomedical, has served as an advisor/consultant to COTA Healthcare, and has served on a grant review panel for the National Comprehensive Cancer Network funded by Taiho Pharmaceutical. M.G. received research funding from Bristol-Myers Squibb, Merck, and Servier. This study was not funded by any of these commercial entities. A.T.C. previously served as a consultant for Bayer Healthcare and Pfizer Inc. This study was not funded by Bayer Healthcare or Pfizer Inc. K.N. has received institutional research funding from Evergrande Group, Genentech, Gilead Sciences, Pharmavite, Revolution Medicines, Tarrex Biopharma, and Trovagene and has served on advisory boards for Array Biopharma, Bayer, 
and Seattle Genetics. The other authors declare that they have no conflicts of interest. The funders had no role in the design of the study; in the collection, analyses, or interpretation of data; in the writing of the manuscript, or in the decision to publish the results.

$\begin{array}{ll}\text { Abbreviations } \\ \text { AJCC } & \begin{array}{l}\text { American Joint Committee on Cancer } \\ \text { confidence interval }\end{array} \\ \text { CI } & \text { CpG island methylator phenotype } \\ \text { CIMP } & \begin{array}{l}\text { formalin-fixed paraffin-embedded } \\ \text { FFPE }\end{array} \\ \text { HR } & \text { hazard ratio } \\ \text { LCM } & \text { laser capture microdissection } \\ \text { LINE-1 } & \begin{array}{l}\text { long interspersed nucleotide element-1 } \\ \text { MSI }\end{array} \\ \text { microsatellite instability } \\ \text { PCR } & \text { polymerase chain reaction } \\ \text { SD } & \text { standard deviation } \\ \text { TNM } & \text { tumor, node, and metastases }\end{array}$

\section{References}

1. Siegel, R.L.; Torre, L.A.; Soerjomataram, I.; Hayes, R.B.; Bray, F.; Weber, T.K.; Jemal, A. Global patterns and trends in colorectal cancer incidence in young adults. Gut 2019, 68, 2179-2185. [CrossRef] [PubMed]

2. Saad El Din, K.; Loree, J.M.; Sayre, E.C.; Gill, S.; Brown, C.J.; Dau, H.; De Vera, M.A. Trends in the epidemiology of young-onset colorectal cancer: A worldwide systematic review. BMC Cancer 2020, 20, 288. [CrossRef] [PubMed]

3. Chung, R.Y.; Tsoi, K.K.F.; Kyaw, M.H.; Lui, A.R.; Lai, F.T.T.; Sung, J.J. A population-based age-period-cohort study of colorectal cancer incidence comparing Asia against the West. Cancer Epidemiol. 2019, 59, 29-36. [CrossRef] [PubMed]

4. Sung, H.; Siegel, R.L.; Rosenberg, P.S.; Jemal, A. Emerging cancer trends among young adults in the USA: Analysis of a population-based cancer registry. Lancet Public Health 2019, 4, e137-e147. [CrossRef]

5. Hofseth, L.J.; Hebert, J.R.; Chanda, A.; Chen, H.; Love, B.L.; Pena, M.M.; Murphy, E.A.; Sajish, M.; Sheth, A.; Buckhaults, P.J.; et al. Early-onset colorectal cancer: Initial clues and current views. Nat. Rev. Gastroenterol. Hepatol. 2020, 17, 352-364. [CrossRef]

6. Stoffel, E.M.; Murphy, C.C. Epidemiology and Mechanisms of the Increasing Incidence of Colon and Rectal Cancers in Young Adults. Gastroenterology 2020, 158, 341-353. [CrossRef] [PubMed]

7. Akimoto, N.; Ugai, T.; Zhong, R.; Hamada, T.; Fujiyoshi, K.; Giannakis, M.; Wu, K.; Cao, Y.; Ng, K.; Ogino, S. Rising incidence of early-onset colorectal cancer-A call to action. Nat. Rev. Clin. Oncol. 2021, 18, 230-243. [CrossRef]

8. Lieu, C.H.; Golemis, E.A.; Serebriiskii, I.G.; Newberg, J.; Hemmerich, A.; Connelly, C.; Messersmith, W.A.; Eng, C.; Eckhardt, S.G.; Frampton, G.; et al. Comprehensive Genomic Landscapes in Early and Later Onset Colorectal Cancer. Clin. Cancer Res. 2019, 25, 5852-5858. [CrossRef]

9. Willauer, A.N.; Liu, Y.; Pereira, A.A.L.; Lam, M.; Morris, J.S.; Raghav, K.P.S.; Morris, V.K.; Menter, D.; Broaddus, R.; MericBernstam, F.; et al. Clinical and molecular characterization of early-onset colorectal cancer. Cancer 2019, 125, 2002-2010. [CrossRef]

10. Nimptsch, K.; Wu, K. Is Timing Important? The Role of Diet and Lifestyle during Early Life on Colorectal Neoplasia. Curr. Colorectal. Cancer Rep. 2018, 14, 1-11. [CrossRef] [PubMed]

11. Liu, P.H.; Wu, K.; Ng, K.; Zauber, A.G.; Nguyen, L.H.; Song, M.; He, X.; Fuchs, C.S.; Ogino, S.; Willett, W.C.; et al. Association of Obesity With Risk of Early-Onset Colorectal Cancer Among Women. JAMA Oncol. 2019, 5, 37-44. [CrossRef] [PubMed]

12. Szyf, M. The early life environment and the epigenome. Biochim. Biophys. Acta 2009, 1790, 878-885. [CrossRef] [PubMed]

13. Ogino, S.; Lochhead, P.; Chan, A.T.; Nishihara, R.; Cho, E.; Wolpin, B.M.; Meyerhardt, J.A.; Meissner, A.; Schernhammer, E.S.; Fuchs, C.S.; et al. Molecular pathological epidemiology of epigenetics: Emerging integrative science to analyze environment, host, and disease. Mod. Pathol. 2013, 26, 465-484. [CrossRef] [PubMed]

14. Feinberg, A.P. The Key Role of Epigenetics in Human Disease Prevention and Mitigation. N. Engl. J. Med. 2018, 378, 1323-1334. [CrossRef] [PubMed]

15. Baba, Y.; Yagi, T.; Sawayama, H.; Hiyoshi, Y.; Ishimoto, T.; Iwatsuki, M.; Miyamoto, Y.; Yoshida, N.; Baba, H. Long Interspersed Element-1 Methylation Level as a Prognostic Biomarker in Gastrointestinal Cancers. Digestion 2018, 97, 26-30. [CrossRef] [PubMed]

16. Antelo, M.; Balaguer, F.; Shia, J.; Shen, Y.; Hur, K.; Moreira, L.; Cuatrecasas, M.; Bujanda, L.; Giraldez, M.D.; Takahashi, M.; et al. A high degree of LINE-1 hypomethylation is a unique feature of early-onset colorectal cancer. PLoS ONE 2012, 7, e45357. [CrossRef] [PubMed]

17. Baba, Y.; Huttenhower, C.; Nosho, K.; Tanaka, N.; Shima, K.; Hazra, A.; Schernhammer, E.S.; Hunter, D.J.; Giovannucci, E.L.; Fuchs, C.S.; et al. Epigenomic diversity of colorectal cancer indicated by LINE-1 methylation in a database of 869 tumors. Mol. Cancer 2010, 9, 125. [CrossRef] [PubMed] 
18. Ogino, S.; Kawasaki, T.; Nosho, K.; Ohnishi, M.; Suemoto, Y.; Kirkner, G.J.; Fuchs, C.S. LINE-1 hypomethylation is inversely associated with microsatellite instability and CpG island methylator phenotype in colorectal cancer. Int. J. Cancer 2008, 122, 2767-2773. [CrossRef]

19. Inamura, K.; Yamauchi, M.; Nishihara, R.; Lochhead, P.; Qian, Z.R.; Kuchiba, A.; Kim, S.A.; Mima, K.; Sukawa, Y.; Jung, S.; et al. Tumor LINE-1 methylation level and microsatellite instability in relation to colorectal cancer prognosis. J. Natl. Cancer Inst. 2014, 106, dju195. [CrossRef]

20. Mima, K.; Nowak, J.A.; Qian, Z.R.; Cao, Y.; Song, M.; Masugi, Y.; Shi, Y.; da Silva, A.; Gu, M.; Li, W.; et al. Tumor LINE-1 methylation level and colorectal cancer location in relation to patient survival. Oncotarget 2016, 7, 55098-55109. [CrossRef]

21. Nishihara, R.; Wu, K.; Lochhead, P.; Morikawa, T.; Liao, X.; Qian, Z.R.; Inamura, K.; Kim, S.A.; Kuchiba, A.; Yamauchi, M.; et al. Long-term colorectal-cancer incidence and mortality after lower endoscopy. N. Engl. J. Med. 2013, 369, 1095-1105. [CrossRef] [PubMed]

22. Haruki, K.; Kosumi, K.; Li, P.; Arima, K.; Väyrynen, J.P.; Lau, M.C.; Twombly, T.S.; Hamada, T.; Glickman, J.N.; Fujiyoshi, K.; et al. An integrated analysis of lymphocytic reaction, tumour molecular characteristics and patient survival in colorectal cancer. Br. J. Cancer 2020, 122, 1367-1377. [CrossRef] [PubMed]

23. Yamauchi, M.; Morikawa, T.; Kuchiba, A.; Imamura, Y.; Qian, Z.R.; Nishihara, R.; Liao, X.; Waldron, L.; Hoshida, Y.; Huttenhower, C.; et al. Assessment of colorectal cancer molecular features along bowel subsites challenges the conception of distinct dichotomy of proximal versus distal colorectum. Gut 2012, 61, 847-854. [CrossRef] [PubMed]

24. Irahara, N.; Nosho, K.; Baba, Y.; Shima, K.; Lindeman, N.I.; Hazra, A.; Schernhammer, E.S.; Hunter, D.J.; Fuchs, C.S.; Ogino, S. Precision of pyrosequencing assay to measure LINE-1 methylation in colon cancer, normal colonic mucosa, and peripheral blood cells. J. Mol. Diagn 2010, 12, 177-183. [CrossRef] [PubMed]

25. Burden, A.F.; Manley, N.C.; Clark, A.D.; Gartler, S.M.; Laird, C.D.; Hansen, R.S. Hemimethylation and non-CpG methylation levels in a promoter region of human LINE-1 (L1) repeated elements. J. Biol. Chem. 2005, 280, 14413-14419. [CrossRef]

26. Ogino, S.; Nosho, K.; Kirkner, G.J.; Kawasaki, T.; Meyerhardt, J.A.; Loda, M.; Giovannucci, E.L.; Fuchs, C.S. CpG island methylator phenotype, microsatellite instability, BRAF mutation and clinical outcome in colon cancer. Gut 2009, 58, 90-96. [CrossRef]

27. Fujiyoshi, K.; Bruford, E.A.; Mroz, P.; Sims, C.L.; O’Leary, T.J.; Lo, A.W.I.; Chen, N.; Patel, N.R.; Patel, K.P.; Seliger, B.; et al. Opinion: Standardizing gene product nomenclature-a call to action. Proc. Natl. Acad. Sci. USA 2021, 118, e2025207118. [CrossRef] [PubMed]

28. Liao, X.; Lochhead, P.; Nishihara, R.; Morikawa, T.; Kuchiba, A.; Yamauchi, M.; Imamura, Y.; Qian, Z.R.; Baba, Y.; Shima, K.; et al. Aspirin use, tumor PIK3CA mutation, and colorectal-cancer survival. N. Engl. J. Med. 2012, 367, 1596-1606. [CrossRef] [PubMed]

29. Benjamin, D.J.; Berger, J.O.; Johannesson, M.; Nosek, B.A.; Wagenmakers, E.J.; Berk, R.; Bollen, K.A.; Brembs, B.; Brown, L.; Camerer, C.; et al. Redefine statistical significance. Nat. Hum. Behav. 2018, 2, 6-10. [CrossRef]

30. Gupta, S.; Harper, A.; Ruan, Y.; Barr, R.; Frazier, A.L.; Ferlay, J.; Steliarova-Foucher, E.; Fidler-Benaoudia, M.M. International trends in the incidence of cancer among adolescents and young adults. J. Natl. Cancer Inst. 2020, 112, 1105-1117. [CrossRef] [PubMed]

31. Siegel, R.L.; Fedewa, S.A.; Anderson, W.F.; Miller, K.D.; Ma, J.; Rosenberg, P.S.; Jemal, A. Colorectal Cancer Incidence Patterns in the United States, 1974-2013. J. Natl. Cancer. Inst. 2017, 109, djw322. [CrossRef]

32. Kneuertz, P.J.; Chang, G.J.; Hu, C.Y.; Rodriguez-Bigas, M.A.; Eng, C.; Vilar, E.; Skibber, J.M.; Feig, B.W.; Cormier, J.N.; You, Y.N. Overtreatment of young adults with colon cancer: More intense treatments with unmatched survival gains. JAMA Surg. 2015, 150, 402-409. [CrossRef] [PubMed]

33. Yeo, H.; Betel, D.; Abelson, J.S.; Zheng, X.E.; Yantiss, R.; Shah, M.A. Early-onset Colorectal Cancer is Distinct From Traditional Colorectal Cancer. Clin. Colorectal. Cancer 2017, 16, 293-299.e296. [CrossRef] [PubMed]

34. Soliman, B.G.; Karagkounis, G.; Church, J.M.; Plesec, T.; Kalady, M.F. Mucinous Histology Signifies Poor Oncologic Outcome in Young Patients With Colorectal Cancer. Dis. Colon Rectum 2018, 61, 547-553. [CrossRef]

35. Mauri, G.; Sartore-Bianchi, A.; Russo, A.G.; Marsoni, S.; Bardelli, A.; Siena, S. Early-onset colorectal cancer in young individuals. Mol. Oncol. 2019, 13, 109-131. [CrossRef]

36. Chen, F.W.; Sundaram, V.; Chew, T.A.; Ladabaum, U. Advanced-Stage Colorectal Cancer in Persons Younger Than 50 Years Not Associated With Longer Duration of Symptoms or Time to Diagnosis. Clin. Gastroenterol. Hepatol. 2017, 15, 728-737.e723. [CrossRef]

37. Stoffel, E.M.; Koeppe, E.; Everett, J.; Ulintz, P.; Kiel, M.; Osborne, J.; Williams, L.; Hanson, K.; Gruber, S.B.; Rozek, L.S. Germline Genetic Features of Young Individuals With Colorectal Cancer. Gastroenterology 2018, 154, 897-905.e891. [CrossRef] [PubMed]

38. Mork, M.E.; You, Y.N.; Ying, J.; Bannon, S.A.; Lynch, P.M.; Rodriguez-Bigas, M.A.; Vilar, E. High Prevalence of Hereditary Cancer Syndromes in Adolescents and Young Adults With Colorectal Cancer. J. Clin. Oncol. 2015, 33, 3544-3549. [CrossRef] [PubMed]

39. Archambault, A.N.; Su, Y.R.; Jeon, J.; Thomas, M.; Lin, Y.; Conti, D.V.; Win, A.K.; Sakoda, L.C.; Lansdorp-Vogelaar, I.; Peterse, E.F.P.; et al. Cumulative Burden of Colorectal Cancer-Associated Genetic Variants Is More Strongly Associated With Early-Onset vs Late-Onset Cancer. Gastroenterology 2020, 158, 1274-1286.e1212. [CrossRef] [PubMed]

40. Gausman, V.; Dornblaser, D.; Anand, S.; Hayes, R.B.; O'Connell, K.; Du, M.; Liang, P.S. Risk Factors Associated With Early-Onset Colorectal Cancer. Clin. Gastroenterol. Hepatol. 2020, 18, 2752-2759. [CrossRef]

41. Rosato, V.; Bosetti, C.; Levi, F.; Polesel, J.; Zucchetto, A.; Negri, E.; La Vecchia, C. Risk factors for young-onset colorectal cancer. Cancer Causes Control 2013, 24, 335-341. [CrossRef] 
42. Nilsson, E.E.; Skinner, M.K. Environmentally Induced Epigenetic Transgenerational Inheritance of Reproductive Disease1. Biol. Reprod. 2015, 93, 1-8. [CrossRef]

43. Rodriguez-Martin, B.; Alvarez, E.G.; Baez-Ortega, A.; Zamora, J.; Supek, F.; Demeulemeester, J.; Santamarina, M.; Ju, Y.S.; Temes, J.; Garcia-Souto, D.; et al. Pan-cancer analysis of whole genomes identifies driver rearrangements promoted by LINE-1 retrotransposition. Nat. Genet. 2020, 52, 306-319. [CrossRef]

44. Iskow, R.C.; McCabe, M.T.; Mills, R.E.; Torene, S.; Pittard, W.S.; Neuwald, A.F.; Van Meir, E.G.; Vertino, P.M.; Devine, S.E. Natural mutagenesis of human genomes by endogenous retrotransposons. Cell 2010, 141, 1253-1261. [CrossRef] [PubMed]

45. Kitkumthorn, N.; Mutirangura, A. Long interspersed nuclear element-1 hypomethylation in cancer: Biology and clinical applications. Clin. Epigenetics 2011, 2, 315-330. [CrossRef] [PubMed]

46. Sunami, E.; de Maat, M.; Vu, A.; Turner, R.R.; Hoon, D.S. LINE-1 hypomethylation during primary colon cancer progression. PLoS ONE 2011, 6, e18884. [CrossRef] [PubMed]

47. Ye, D.; Jiang, D.; Li, Y.; Jin, M.; Chen, K. The role of LINE-1 methylation in predicting survival among colorectal cancer patients: A meta-analysis. Int. J. Clin. Oncol. 2017, 22, 749-757. [CrossRef]

48. Ogino, S.; Nosho, K.; Kirkner, G.J.; Kawasaki, T.; Chan, A.T.; Schernhammer, E.S.; Giovannucci, E.L.; Fuchs, C.S. A cohort study of tumoral LINE-1 hypomethylation and prognosis in colon cancer. J. Natl. Cancer Inst. 2008, 100, 1734-1738. [CrossRef] [PubMed]

49. Lou, Y.T.; Chen, C.W.; Fan, Y.C.; Chang, W.C.; Lu, C.Y.; Wu, I.C.; Hsu, W.H.; Huang, C.W.; Wang, J.Y. LINE-1 Methylation Status Correlates Significantly to Post-Therapeutic Recurrence in Stage III Colon Cancer Patients Receiving FOLFOX-4 Adjuvant Chemotherapy. PLoS ONE 2014, 10, e0123973. [CrossRef] [PubMed]

50. Swets, M.; Zaalberg, A.; Boot, A.; van Wezel, T.; Frouws, M.A.; Bastiaannet, E.; Gelderblom, H.; van de Velde, C.J.; Kuppen, P.J. Tumor LINE-1 Methylation Level in Association with Survival of Patients with Stage II Colon Cancer. Int. J. Mol. Sci. 2016, 18, 36. [CrossRef] [PubMed]

51. Hur, K.; Cejas, P.; Feliu, J.; Moreno-Rubio, J.; Burgos, E.; Boland, C.R.; Goel, A. Hypomethylation of long interspersed nuclear element-1 (LINE-1) leads to activation of proto-oncogenes in human colorectal cancer metastasis. Gut 2014, 63, 635-646. [CrossRef] [PubMed]

52. Ehrlich, M. DNA hypermethylation in disease: Mechanisms and clinical relevance. Epigenetics 2019, 14, 1141-1163. [CrossRef] [PubMed]

53. Issa, J.P. CpG island methylator phenotype in cancer. Nat. Rev. Cancer 2004, 4, 988-993. [CrossRef] [PubMed]

54. Ogino, S.; Goel, A. Molecular classification and correlates in colorectal cancer. J. Mol. Diagn. 2008, 10, 13-27. [CrossRef] [PubMed]

55. Wolf, A.M.D.; Fontham, E.T.H.; Church, T.R.; Flowers, C.R.; Guerra, C.E.; LaMonte, S.J.; Etzioni, R.; McKenna, M.T.; Oeffinger, K.C.; Shih, Y.T.; et al. Colorectal cancer screening for average-risk adults: 2018 guideline update from the American Cancer Society. CA Cancer J. Clin. 2018, 68, 250-281. [CrossRef]

56. Shaukat, A.; Kahi, C.J.; Burke, C.A.; Rabeneck, L.; Sauer, B.G.; Rex, D.K. ACG Clinical Guidelines: Colorectal Cancer Screening 2021. Am. J. Gastroenterol. 2021, 116, 458-479. [CrossRef]

57. Ogino, S.; Nowak, J.A.; Hamada, T.; Phipps, A.I.; Peters, U.; Milner, D.A., Jr.; Giovannucci, E.L.; Nishihara, R.; Giannakis, M.; Garrett, W.S.; et al. Integrative analysis of exogenous, endogenous, tumour and immune factors for precision medicine. Gut 2018, 67, 1168-1180. [CrossRef]

58. Ogino, S.; Chan, A.T.; Fuchs, C.S.; Giovannucci, E. Molecular pathological epidemiology of colorectal neoplasia: An emerging transdisciplinary and interdisciplinary field. Gut 2011, 60, 397-411. [CrossRef] [PubMed]

59. Ogino, S.; Nowak, J.A.; Hamada, T.; Milner, D.A., Jr.; Nishihara, R. Insights into Pathogenic Interactions Among Environment, Host, and Tumor at the Crossroads of Molecular Pathology and Epidemiology. Annu. Rev. Pathol. 2019, 14, 83-103. [CrossRef] [PubMed]

60. Hughes, L.A.E.; Simons, C.; van den Brandt, P.A.; van Engeland, M.; Weijenberg, M.P. Lifestyle, Diet, and Colorectal Cancer Risk According to (Epi)genetic Instability: Current Evidence and Future Directions of Molecular Pathological Epidemiology. Curr. Colorectal. Cancer Rep. 2017, 13, 455-469. [CrossRef]

61. Carr, P.R.; Alwers, E.; Bienert, S.; Weberpals, J.; Kloor, M.; Brenner, H.; Hoffmeister, M. Lifestyle factors and risk of sporadic colorectal cancer by microsatellite instability status: A systematic review and meta-analyses. Ann. Oncol. 2018, 29, 825-834. [CrossRef] [PubMed]

62. Wang, S.T.; Cui, W.Q.; Pan, D.; Jiang, M.; Chang, B.; Sang, L.X. Tea polyphenols and their chemopreventive and therapeutic effects on colorectal cancer. World J. Gastroenterol. 2020, 26, 562-597. [CrossRef] [PubMed]

63. Wang, R.; Chen, M.; Ye, X.; Poon, K. Role and potential clinical utility of ARID1A in gastrointestinal malignancy. Mutat. Res. Rev. Mutat. Res. 2021, 787, 108360. [CrossRef]

64. Yang, A.S.; Estécio, M.R.; Doshi, K.; Kondo, Y.; Tajara, E.H.; Issa, J.P. A simple method for estimating global DNA methylation using bisulfite PCR of repetitive DNA elements. Nucleic Acids Res. 2004, 32, e38. [CrossRef] 